Liquid column chromatography

Liquid Column Chromatography: A Survey of Modern Techniques and Applications. (Journal of Chromatography Library, Volume 3.) Edited by Zdenek Deyl, Karel Macek and Jaroslav Janak. Pp. xxi+1,175. (Elsevier Scientific: Amsterdam, London and New York, 1975.) Dfl. 290; \$120.95.

ThIs collaborative 1,120-page volume, almost entirely the work of a group of Czech authors, is undoubtedly the most comprehensive account of the subject currently available. It is divided into four main sections: theoretical aspects of liquid chromatography, techniques of liquid chromatography, practice of liquid chromatography, and applications. The first three sections are general and make up about one-third (413 pages) of the book, whereas the application of these methods to specialised classes of compounds make up the remaining two-thirds.

The general theoretical section is in some respects the least successful part of the book, as in my opinion too much attention is paid to mathematical rigour and insufficient space (only some 10 pages) to the factors which influence zone resolution in practical chromatography. The short section on the thermodynamics of partition and adsorption processes by Novak, however, is excellent. It is rather disappointing to find the old but fallacious concept of a direct relation between solute molecular weight and retention volume adopted in all the sections on gel permeation (or molecular-sieve) chromatography.

The second and third sections on the techniques and practice of liquid column chromatography are excellent. The editors explain that they have decided, wisely in my view, to give equal prominence to the "classical" methods of liquid chromatography and to the newer techniques of high efficiency, high pressure chromatography, especially as the former still constitute $90 \%$ of published methods. The techniques section is essentially confined to instrumentation and materials (mobile and stationary phases) for all the fundamental subgroups of chromatography, adsorption, partition, ion-exchange and the exciting new development of affinity chromatography. The chapters (8 and 11) on instrumentation and operation of high efficiency, high pressure systems compare favourably with some of the specialised works on the subject. Chapter 9 on stationary phases and chapter 10 on mobile phases are particularly valuable, and are admirably complemented by the corresponding chapters 13 and 14 in the practice section. These sections include extensive tables of the properties of the relevant stationary and support phases, as well as those of mobile phase solvents. The criteria for choice of phase, and packing, conditioning and operation of column receive special attention. The sections throughout the book contributed by $\mathrm{Dr} \mathrm{O}$. Mikes on ion-exchange chromatography and by Mrs J. Turkova on affinity chromatography must be specially commended.

The special section of the book gives detailed recommendations for the chromatographic separation of a variety of chemical compounds ranging through hydrocarbons, alcohols through carbohydrates, lipids, amino acids peptides and proteins, nucleic acids and their constituents to some specialised classes of organic compounds such as vitamins, antibodies and pesticides, to cells and subcellular particles, and finally to inorganic compounds.

It is always difficult to assess the value of such large specialised sections

\section{Dynamics of galaxies}

The Formation and Dynamics of Galaxies-Proceedings of IAU Symposium No 58, Canberra, Australia, 1973. Edited by J. R. Shakeshaft. Pp. $\mathrm{xv}+441$. (Reidel : Dordrecht, Holland; Boston, Massachusetts, 1974.) Cloth Dfl. 135, \$54.00; paper Dfl. 100, $\$ 38.50$.

THE publications which arise from IAU symposia are among the most valuable books in any library used by astronomical researchers, and the present round is appearing with commendable speed, given the usual timescale for publication of proceedings volumes. Little has happened since August 1973 to reduce the value of these contributions from the leading lights in cosmology and cosmogeny who were then assembled in Canberra; on the other hand, by the very nature of the book many of the contributions have a familiar ring about them.

The editor has rearranged the record of discussion to keep comments near the papers to which they apply, and has also changed the order of presentation of the contributions to fit a more logical pattern than the exigencies of the Canberra timetable allowed. But he has not attempted to provide an overview of the state of the art, and in the field of galaxy formation more than any other in astronomy (except, perhaps, cosmology) a chapter providing such guidance would be invaluable to readers not already thoroughly in any book, as only a small portion of the material presented will be of any value or even interest to any particular reader, and also such material is more likely to become outdated than general sections. I believe that the best solution is a series of well-documented and indexed tables which will guide the interested reader into the literature of his own subject, without increasing the bulk (and cost) of the book with specialised material of no interest to him.

Among the best of these special chapters are 32 on amino acids by Zmrhal, Heathcote and Washington, 34 on peptides by Khluh, 35 and 36 on proteins by Prusik and Mikes, and 37 and 38 on nucleic acids and their constituents by Zadrazil. These are virtually monographs on their subjects.

In summary, this is by far the best book on liquid column chromatography to appear to date. It is excellently produced, but it is unfortunate that its price will render it inaccessible to many departmental libraries.

C. J. O. R. Morris

familiar with the state of play.

It is no longer very unlikely that non-specialists will take an interest in the areas of study covered by this book, because the high energy astrophysical aspects, in particular, are receiving increasing attention from theoretical physicists who have a background in non-astronomical specialities. But even the congnoscenti have a real problem in distinguishing the wood of a broadly based understanding of how the matter in the Universe got into its present overall state from the many trees of particular theories which explain observations of particular objects or groups of objects without being fitted satisfactorily to the overall pattern.

If you are one of those theorists beavering away on some aspect of the overall problem, then you will find this volume useful not only in providing other models for comparison but in giving some of the observational constraints which must be met by all models. In addition, most active modellers will gain some comfort from the present state of the art; regrettable though it may be to those who treasure the idea of the Universe as a basically simple place, any overview of the work described here must surely lead to the conclusion that both the extreme casesexpansion or outbursts from nuclei, and collapse or condensation of gas clouds-play a part in the real Universe. And there are the various cases of interactions between galaxies to confuse the picture. John Gribbin 